\title{
Proposition D'une Demarche De Gestion Pour Reduire Les Risques De Conduite Des Projets D'implementation Des ERP Logistique
}

\author{
Abdillah Kadouri \\ Professeur Habilité, Ecole Nationale de Commerce et de Gestion, \\ Université Ibn Tofail-Kenitra \\ Anouar Ammi \\ Docteur en Sciences Economiques - Expert en Gestion des Projets de \\ Systèmes d'information, Université Ibn Tofail-Kenitra
}

doi: 10.19044/esj.2016.v12n16p474 URL:http://dx.doi.org/10.19044/esj.2016.v12n16p474

\begin{abstract}
The logistics activity exercise is in fact submitted to several constraints related to physical, financial and information flows; it also requires operational expenses optimization, compliance with performance indicators and processes control.

Being structured in different integrated modules, the ERPs provide operational, tactical and strategic planning features that allow the company to balance its supply and demand plans.

Although project management practices are considered effective nowadays, many companies are still struggling to implement these ERPs and automate the various logistics processes: indeed the situation of such projects becomes uncontrollable or they are abandoned.

It is therefore appropriate to consider, in this article, the main risks that may arise during the logistics integration in the ERPs; then try to explain the most adequate governance to implement in order to reduce their impact.
\end{abstract}

Keywords:Risk,Project Management, ERP, Logistics, Governance.

Résumé

L'exercice de l'activité de la logistique est soumis à plusieurs contraintes relatives aux flux physiques, financiers et d'information ; il exige par ailleurs l'optimisation des charges fonctionnelles, le respect des indicateurs de performance et la maitrise des processus.

Étant structurés en différents modules intégrés, les ERPs proposent des fonctionnalités de planification opérationnelle, tactique et stratégique qui permettent à l'entreprise d'équilibrer ses plans de l'offre et de la demande. 
Or, et malgré l'avancement des pratiques de gestion des projets, plusieurs entreprises peinent à implémenter ces progiciels et à automatiser les différents processus de logistique, les projets menés subissent des dérapages significatifs ou sont abandonnés.

Il convient donc de s'interroger, dans le présent article, sur les principaux risques pouvant surgir lors de l'intégration des processus de la logistique dans les ERPs ; puis d'essayer d'expliciter la démarche de gouvernance la plus appropriée pour en réduire l'impact.

Mots clés :Risques, Gestion de Projets, ERP,Logistique, Gouvernance.

\section{Introduction générale}

Afin de disposer d'indicateurs de performance de leurs opérations, les entreprises doivent traiter une multitude de données émanant de l'ensemble des intervenants, à savoir les fournisseurs, les clients ainsi que les entités internes de l'entreprise. Les systèmes d'aide à la décision apportent une solution à cette problématique.

Les $\mathrm{ERP}^{1}$ fournissent des outils qui permettent de gérer la globalité de la chaîne logistique, ils dotent l'utilisateur final d'une interface qui lui assure une vue globaledes volets de la chaîne logistique, ainsi que le contrôle des processus de planification et d'ordonnancement.

Avant d'être mis en exploitation, l'ERP nécessite généralement des cycles de mise en œuvre longs et coûteux. Si les bénéfices associés sont significatifs, les risques d'échec de réalisation sont importants, plusieurs projets ont été abandonnés et ont entrainé des pertes colossales pour les entreprises, et ce à l'instar de :

\begin{tabular}{|l|c|c|}
\hline \multicolumn{1}{|c|}{ Entreprise } & Logiciel & Impact \\
\hline TRI VALLEY GROWERS & ORACLE & $20 \mathrm{MS}$ \\
\hline HERSHEY FOODS & SAP & $150 \mathrm{MS}$ \\
\hline
\end{tabular}

Les risques du projet sont principalement dus à l'incertitude et à la complexité de l'environnement dans lequel il se déroule et leur impact dépendra des conséquences sur les objectifs du projet. Ce papier s'intéresse à proposer la démarche de gestion la plus appropriée pour éviter l'échec de la mise en place de l'ERP.

1 Entreprise ressources planning. 


\section{Risques d'implémentation des ERP au sein des entreprises.}

\subsection{Les domaines ${ }^{2}$ traditionnellement couverts par les ERP}

L'informatique de gestion cible tous les domaines des organisations, qu'ils soient fonctionnels, décisionnels ou stratégiques. Les entreprises procèdent à l'intégration par le biais de progiciels de gestion intégrée de leurs processus métiers, et ce afin d'éliminer les cloisonnements qui freinent leur développement.

Le concept d'ERP ${ }^{3}$ existe depuis les débuts de la Gestion de Production Assistée par Ordinateur ${ }^{4}$, c'est un sous-ensemble du système $\mathrm{d}^{\prime}$ information qui permet ${ }^{\mathbf{5}}$ une gestion de plusieurs domaines de l'entreprise par des modules intégrés susceptibles d'assurer une synchronisation et corrélation des processus, il permet des adaptations rapides aux règles de fonctionnement de l'organisation interne de l'entreprise et des règles imposées par le marché.

Un progiciel ERP induit généralement une réponse rapide aux besoins des entreprises, il est basé sur la construction des applications informatiques de l'entreprise sous forme de modules indépendants qui partagent une base de données commune, et permettent la communication des données entre les applications.

Les applications qui sont d'habitude concernées par les noyaux des ERP ne recouvrent que des domaines classiques de la gestion de grandes entreprises. Les éditeurs n'ont intérêt à développer des ERP qu'après la vérification en simultanée de la stabilité des règles de gestion imposées par la législation, et des méthodes de gestion et d'organisation utilisées dans un secteur pour la même famille de métiers à travers le monde. En effet, selon le type d'activité, il existe des méthodes facilement adaptables ${ }^{6}$.

\subsection{Développement des pratiques de gestion de projets :}

Le développement des disciplines de gestion de projets n'est pas récent, en effet depuis des milliers d'années, les hommes se sont organisés pour inventer et réaliser des ouvrages, ils ont mis en place plusieurs techniques pour passer du monde de l'imagination au monde réel.

2 Etude «Retour d'expérience ERP» rédigée par Pierre Dubarry (Péchiney) et Virginie Bauvais (Cigref) en Septembre 1999.

3 Acronyme anglais qui désigne le système intégré de gestion d'entreprise

4 (GPAO).

5 Jean-Louis LEQUEUX/ Manager avec les ERP 2008/Architecture Orientée Services (SOA)/ 3ème édition/Éditions d'Organisation, Groupe Eyrolles, 61, bd Saint-Germain, 75240 Paris cedex 05.

6Par exemple, pour la production en série, on peut choisir un modèle d'organisation permettant de gérer en zéro stock. 
Toutefois, la gestion de projet en tant que discipline structurée n'a commencé à instaurer ces leviers qu'à partir de la deuxième guerre mondiale qui a obligé les pays à accélérer le rythme et à améliorer la qualité des projets réalisés. A cet effet, et à partir de 1960, plusieurs associations professionnelles ont diffusé les outils de gestion utilisés. Le management de projet est devenu un champ structuré de connaissances à partir de quatre sources:

- Les sciences de l'ingénieur, qui englobent les méthodes permettant de réaliser et de réussir des projets civils ou militaires.

- La conduite des grands projets, qui a amené les pouvoirs publics à s'interroger sur les problématiques de l'évaluation et de la préparation des décisions.

- Les opérations de développement socio-économique qui ont permis d'accumuler des savoir-faire techniques, économiques, sociologiques et écologiques.

- La gestion de l'innovation, qui est devenue progressivement un enjeu concurrentiel majeur pour les entreprises.

Plusieurs standards ont ainsi vu le jour, la méthodologie à utiliser doit être adaptée au contexte et à la nature de chaque projet, les plus utilisés ${ }^{7}$ sont :

\begin{tabular}{|l|c|c|c|}
\hline & PMBOK & PRINCE2 $^{\mathbf{2}}$ & CDP $^{\mathbf{9}}$ \\
\hline Propriétaire & PMI $^{\mathbf{1 0}}$ & OGC $^{\mathbf{1 1}}$ & AFITEP $^{\mathbf{2}}$ \\
\hline Origine & USA & UK & France \\
\hline $\mathbf{1}^{\text {ere }}$ version & 1987 & 1989 & 1945 \\
\hline Utilisation & 125 pays & UK, Europe du nord et Australie & 50 pays \\
\hline
\end{tabular}

Tableau 1 Quelques standards de Gestion de Projets

Les institutions professionnelles ${ }^{\mathbf{1 3}}$ ont joué un rôle primordial dans la diffusion du modèle instrumental de gestion de projet. Créé aux États-Unis en 1969, le PMI est une association qui rassemble et organise les professionnels de gestion de projets dans le monde entier, son modèle ${ }^{\mathbf{1 4}} \mathrm{de}$ gestion s'appuie sur un arsenal sophistiqué de méthodes et d'outils.

7 Source : Guide des certifications SI : comparatif, analyse et tendances ITIL.

8 Acronyme anglais de « Projects in controlled environnements ».

9 Certification en direction de projets.

10Project management institute.

11Office of government commerce.

12Association francophone de management de projets. Site : http://www.afitep.org/.

13 Le PMI a unifié les pratiques de gestion quel que soit le secteur d'activité. 


\subsection{Les facteurs clés de réussite de projets}

En 1998, un sondage réalisé par Clinton et Beth auprès de 150 gestionnaires en ERP a démontré que la technologie n'est pas la première cause d'échec. Les échecs seraient souvent liés à une mauvaise gestion du projet, aux changements des besoins d'affaires durant le projet et au manque de support des gestionnaires fonctionnels.

Certains auteurs ont tenté de délimiter les facteurs clés de succès de conduite de projets, (Belassi et Tukel 1996) les ont classé en quatre dimensions relatives au périmètre couvert, à l'équipe de projet, à l'organisation des interactions au sein du projet, ainsi qu'à l'impact des facteurs externes.

Terry Cooke-Davies a démontré en 2002 à travers son étude «The "real" successfactors on projects » qu'en plus de éléments de délais, de coût et de qualité, la complexité et le positionnement adapté selon qu'on est utilisateur final ou directeur de projet sont autant d'éléments qui permettent de définir ces facteurs.

Ces chercheurs ont ainsi proposé des listes plus au mois exhaustives de ces facteurs clés de succès. Des facteurs tels que la planification, les ressources humaines, la communication et le soutien de la direction générale revêtent un caractère universel. Toutefois, il est à noter que ces éléments ne sont pas similaires dans l'ensemble des projets.

Dans la continuité de ces recherches ${ }^{15}$, Jugdev et Müller ${ }^{16}$ ont présenté 1 'historique ${ }^{17}$ des facteurs clés de succès, ils ont démontré l'existence de différentes listes et de modèles dans la littérature de la gestion de projet depuis les années 1980. La liste la plus connue est celle de Slevin et Pinto $^{18}$ qui propose une base de dix facteurs relatifs à la mission du projet, au soutien de la direction générale, à la planification et les échéanciers, à l'écoute des clients, au personnel, aux tâches techniques, à l'approbation du client, au pilotage et la rétroaction, à la communication et à la gestion des problèmes.

Parr et $\mathrm{Al}$ ont classé ces facteurs de succès en quatre principales catégories ${ }^{19}$ :

- La première est relative aux déterminants de succès des systèmes d'information, et ce à l'instar du rôle des usagers, la disponibilité des ressources qualifiées, la nomination d'un sponsor ainsi que le soutien de la direction générale.

15 Westerveld, 2003.

162005.

17 Source : XVIème Conférence Internationale de Management Stratégique Juin 2007. La recherche sur le succès des projets : approche universelle ou contingente?

181986. 
- La deuxième concerne les déterminants de succès des progiciels, tels que l'engagement de la direction générale, l'optimisation des processus d'implantation, la gestion rigoureuse du projet, les critères de choix du progiciel.

- La troisième catégorie est relative aux techniques de gestion du projet d'implantation des ERP tels la planification, la qualification de l'équipe projet, la communication ainsi que le suivi rigoureux d'avancement.

- La quatrième catégorie concerne l'implantation même des ERP, à l'instar de l'impact de leur mise en service sur la structure et sur la réingénierie des processus.

\subsection{La gestion des risques :}

Dans la littérature, on retrouve les termes d'aléas, de risque, d'incident ou d'incertitude sans pour autant trouver un consensus sur la définition du terme risque, toutefois; toutes les définitions se concentrent sur le fait qu'il s'agit de la survenue d'un évènement qui entravera l'atteinte des objectifs du projet.

Le mot « risque » serait apparu en 1557 dans un traité d'Henri Estienne $^{20}$ comme étant un mot féminin, Il est finalement devenu un mot masculin au XVIIe siècle dans L'impromptu de Versailles de Molière ${ }^{21}$.Le tableau ci-dessous présente quelques définitions :

\begin{tabular}{|c|c|c|}
\hline Auteur & Domaine & Définition \\
\hline AFITEP & Projet & $\begin{array}{l}\text { L'éventualité qu'un projet ne se déroule pas conformément aux objectifs } \\
\text { souhaités. }\end{array}$ \\
\hline AFNOR & Projet & $\begin{array}{l}\text { Evénement dont l'apparition n'est pas certaine et dont la manifestation est } \\
\text { susceptible d'affecter les objectifs du projet. }\end{array}$ \\
\hline $\mathrm{ISO}^{22}$ & Projet & $\begin{array}{l}\text { Couple le risque aux objectifs de l'organisation : « Le risque est l'effet de } \\
\text { l'incertitude sur les objectifs ». }\end{array}$ \\
\hline PMI & Projet & $\begin{array}{l}\text { Mesure de la probabilité d'un événement indésirable qui peut avoir un impact } \\
\text { négatif ou positif sur l'atteinte des objectifs. }\end{array}$ \\
\hline $\mathrm{COSO} 2004^{23}$ & $\begin{array}{l}\text { Contrôle } \\
\text { interne }\end{array}$ & $\begin{array}{l}\text { La possibilité de production d'un évènement qui impacte l'atteinte des } \\
\text { objectifs. }\end{array}$ \\
\hline OGC & Projet & $\begin{array}{l}\text { évènement qui impose aux gestionnaires d'adopter une gestion efficace pour } \\
\text { en réduire l'impact sur le projet. }\end{array}$ \\
\hline $\mathrm{ACN}^{24}$ & Gestion & $\begin{array}{l}\text { Mesure de probabilité et de gravité d'un effet sur la santé, les biens ou } \\
\text { l'environnement. }\end{array}$ \\
\hline
\end{tabular}

Tableau 2 Définition de La Notion de Risque

20Estienne 1578, (1531-1598), il était, philologue, helléniste et humaniste.

21 (1663) (Lannoy 2008)).Ton argent court un risque/ Un dépositaire ne court point de risque

22 Guide 73 - Vocabulaire du management du risque.

23Référentiel de contrôle interne défini par le Committee Of Sponsoring Organizations of the Treadway Commission.

24Association canadienne de normalisation : organisme qui agrée et diffuse les normes au Canada. 
D'autres auteurs se sont intéressés à définir le terme, GIARD ${ }^{25}$ annonce que le risque est: «La possibilité qu'un projet ne s'exécute pas conformément aux prévisions de date d'achèvement, de coûts et de spécifications». CHAPMAN annonce que les risques et les incertitudes sont des termes négatifs mais qui devraient être perçus comme des opportunités et facteurs de réussite.

La survenue des événements imprévisibles peut impacter l'atteinte des objectifs fixés dans les organisations. Ces événements qui ont un impact négatif sur les systèmes ${ }^{26}$ sont suivis et étudiés par la science de la cindynique ${ }^{27}$ qui a mis certaines notions en place :

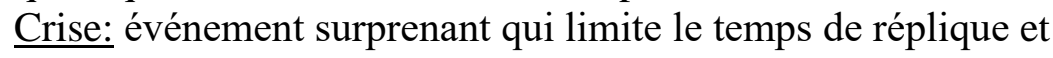
menace les objectifs.

Incident: perturbation d'une composante, d'une unité ou d'un soussystème d'un système.

Risque: aléas dont la survenance l'empêche d'atteindre ses objectifs. Les variables ${ }^{28}$ de risque fréquemment rencontrées sont d'ordre politique, technique, environnemental ou financier.

\subsection{Méthodes de gestion des risques :}

\subsection{1 : Méthodes $^{29}$ TRAM, ARAMIS, RISKMAN pour la gestion des risques.}

Il existe plusieurs méthodes universelles de gestion de risque dans divers domaines, quelques-unes sont présentées dans le tableau suivant :

\begin{tabular}{|l|l|}
\hline Auteur & Domaine couvert \\
\hline AMDEC $^{\mathbf{3 0}}$ & Sûreté de fonctionnement et gestion de la qualité. \\
\hline APR $^{\mathbf{3 1}}$ & Généraliste et couvre plusieurs secteurs d'activités. \\
\hline HACCP $^{\mathbf{3 2}}$ & Sécurité dans la chaîne alimentaire. \\
\hline RRHI $^{\mathbf{3}}$ & Sécurité dans l'industrie chimique. \\
\hline TRAM $^{\mathbf{3 4}}$ & Risques d'une machine ou d'un système dans le domaine technique. \\
\hline ARAMIS & Prévention d'accidents majeurs. \\
\hline RISKMAN & Risk-management. \\
\hline
\end{tabular}

Tableau 3 méthodes universelles de gestion de risque

25Vincent Giard 1995.

26 Projets, entreprises, système naturel.....

27Science du danger.

28Liste non exhaustive.

29 Thèse: «proposition d'une méthode de management des risques multi-projets : application à l'institut Curie-Hôpital », présentée par : Simone Teresinha DA VEIGA le 0312-2012 à l'école nationale d'arts et métiers Paris Tech.

30Analyse des Modes de Défaillances, de leurs Effets et de leur Criticité.

31Analyse Préliminaire des Risques

32HazardAnalysisCritical Control Point

33 Chemical Runaway Reaction Hazard Index)

34Développée en 1998 par Klein et Cork

35Accidental Risk analyse Methodology for Industries 
On peut citer quelques méthodologies qui ont contribué significativement au développement des processus de management des risques.

\section{A- Méthode TRAM:}

La démarche intégrative TRAM préconise d'utiliser des méthodes de qualification adaptées à chaque typologie de risque au lieu de proposer une méthode de mesure universelle. Elle se base sur les étapes suivantes :

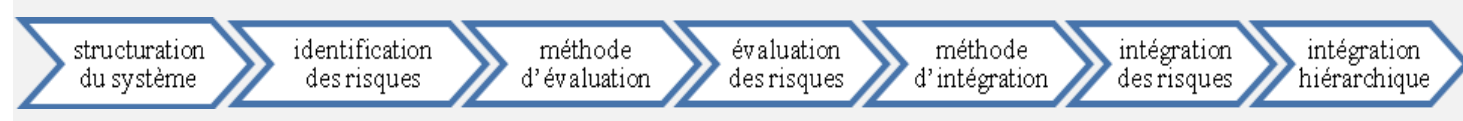

Figure 1 Processus TRAM de Gestion des Risques

Lors de l'étape de l'identification de la méthode d'évaluation, TRAM propose d'évaluer chaque risque par une méthode spécifique qui dépend de ses caractéristiques; elle propose deux techniques ${ }^{\mathbf{3 6}}$ de décomposition et d'évaluation par comparaison pour identifier les risques. De même, elle vise principalement à normaliser les impacts des risques, condition qualifiée de sine qua none au tri des risques par rapport à leurs gravités et probabilités.

\section{B- Méthode RISKMAN :}

La méthode RISKMAN ${ }^{37}$ propose un processus de management des risques identique aux référentiels existants composé des phases d'identification, d'évaluation, de traitement et de suivi des risques, et préconise par contre deux principales règles de gestion relatives à la spécification des catégories de risques et à la mesure de leur impact.

A cet effet; Chaque risque ne doit appartenir qu'à une seule des douze catégories de risques métiers suivants: Stratégie - Marketing Contrats - Finance - Planification de projets- Contenu - Processus - Produits - Organisation - Maintenance - Exploitation - Evénements externes à l'organisation.

De même, RISKMAN stipule qu'il existe une forte corrélation entre les risques due à l'impact de l'occurrence d'un risque sur l'apparition d'autres nouveaux risques. Cet impact doit être correctement évalué, cette corrélation est illustrée par le fait qu'un risque sans impact financier mènera à la production d'un ou de plusieurs risques avec impact financier.

36Klein et Cork 1998.

37Développée entre 1993 et 1996 dans le cadre du projet EUREKA. 


\section{C- Méthode ARAMIS :}

La méthodologie ARAMIS, issue d'un projet ${ }^{\mathbf{3 8}}$ européen de recherche lancé de 2002 à 2004, positionne le risque entre ses causes et son impact. Elle propose d'étudier les scénarios d'accident sous forme de nœud papillon $^{\mathbf{3 9}}$ et de définir des limites de protection pour maitriser les scénarios identifiés.

ARAMIS définit le risque comme la combinaison de la probabilité de survenue d'un phénomène dangereux et de l'événement majeur correspondant, de son ampleur, ainsi que de la vulnérabilité du territoire exposé. L'un de ses principaux apports est relatif à la probabilité d'occurrence des risques situé à gauche dans le « nœud papillon » au niveau de l'arbre de défaillance. L'approche est illustrée dans la figure ci-dessous :

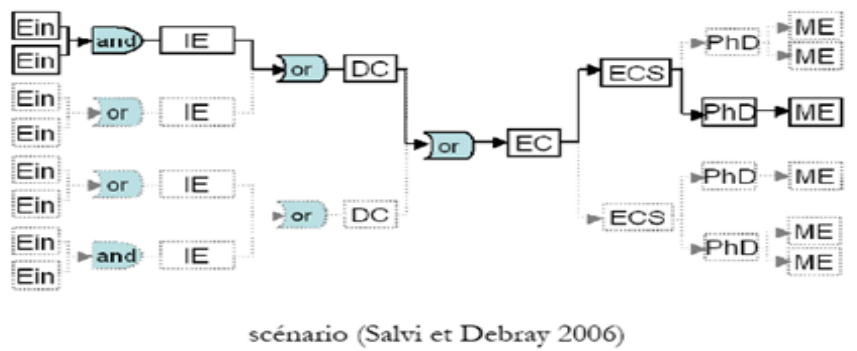

\begin{tabular}{|l|l|}
\hline Abréviation & Correspondance \\
\hline PhD & Phénomènc Dangcrcux. \\
\hline $\mathrm{ME}$ & Evénement Majeur. \\
\hline $\mathrm{EC}$ & Evénement Critique. \\
\hline Ein & Evènements indésirables. \\
\hline $\mathrm{DC}$ & Causes Directes. \\
\hline $\mathrm{ECS}$ & Evènements Critiques Secondaires. \\
\hline
\end{tabular}

Figure 2 Approche ARAMIS

ARAMIS évoque la notion d'arbre de défaillances illustré au niveau de l'onglet gauche du nœud papillon pour identifier les sources d'un évènement critique. Le regroupement d'évènements indésirables entraîne à son tour la survenue d'un autre évènement indésirable, chose qui entraine la survenue d'un évènement critique qui pourra engendrer des évènements critiques secondaires qui risquent de donner lieu à des phénomènes dangereux.

\subsubsection{Le processus de management des risques selon le PMBOK.}

Le management des risques du projet, basé sur des éléments d'entrée, d'outils, de techniques et d'éléments de sortie comprend les processus illustrés dans la figure suivante :

38Le projet visait à développer une nouvelle méthodologie d'évaluation des risques qui répond aux exigences de la directive Seveso II pour remplacer les approches purement probabilistes de l'évaluation des risques.

39CE concept a été initialement développé par Shell pour représenter les étapes de gestion de risques dans une installation. 


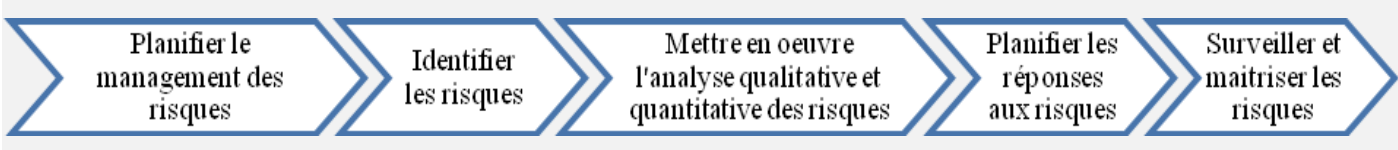

Figure 3 Processus PMBOK de Gestion des Risques

Le PMBOK définit des stratégies distinctes pour gérer les menaces et les opportunités :

\begin{tabular}{|c|c|c|c|}
\hline \multicolumn{2}{|r|}{ Menaces } & \multicolumn{2}{|r|}{ Opportunités } \\
\hline Stratégie & Objectif & Stratégie & Objectif \\
\hline Éviter & $\begin{array}{l}\text { Eliminer la menace, isoler ou } \\
\text { l'objectif qui se trouve menacé. }\end{array}$ & Exploiter & $\begin{array}{l}\text { Peut être choisie pour des } \\
\text { risques à impacts positifs. }\end{array}$ \\
\hline Transférer & $\begin{array}{l}\text { Détourner le risque vers un autre } \\
\text { intervenant. }\end{array}$ & Partager & $\begin{array}{l}\text { Attribuer la responsabilité } \\
\text { à une autre partie } \\
\text { prenante. }\end{array}$ \\
\hline Atténuer & \begin{tabular}{llr} 
réduire la & \multicolumn{2}{l}{ probabilité en } \\
appliquant & des & mesures \\
préventives. & &
\end{tabular} & $\begin{array}{l}\text { Améliore } \\
\mathrm{r}\end{array}$ & $\begin{array}{l}\text { Accroître les impacts } \\
\text { positifs d'une opportunité. }\end{array}$ \\
\hline Accepter & $\begin{array}{l}\text { accepter le risque suite à } \\
\text { l'impossibilité de contournement. }\end{array}$ & Accepter & $\begin{array}{l}\text { Elle consiste à en profiter } \\
\text { dès sa concrétisation. }\end{array}$ \\
\hline
\end{tabular}

Tableau 4 Stratégies PMBOK de Gestion des Menaces et des Opportunités

Les objectifs des livrables de ce processus sont synthétisés dans le tableau suivant :

\begin{tabular}{|l|l|}
\hline \multicolumn{1}{|c|}{ Livrable } & \multicolumn{1}{c|}{ Objectif } \\
\hline $\begin{array}{l}\text { Plan de management } \\
\text { des risques }\end{array}$ & $\begin{array}{l}\text { Définit l'approche de gestion de risques, les rôles et périmètres de } \\
\text { responsabilité de l'équipe, l'affectation du budget, la fréquence des } \\
\text { opérations de suivi et de contrôle, la catégorisation des risques et } \\
\text { l'estimation de leur probabilité d'occurrence et de leur impact. }\end{array}$ \\
\hline Registre des risques & $\begin{array}{l}\text { Contient les risques identifiés et les traitements à y réserver, il doit } \\
\text { être mis à jour régulièrement. }\end{array}$ \\
\hline $\begin{array}{l}\text { Mises à jour des plans } \\
\text { et des documents }\end{array}$ & $\begin{array}{l}\text { Les décisions prises pour la gestion des risques doivent être } \\
\text { enregistrées et documentées quelle que soit la stratégie déployée. }\end{array}$ \\
\hline $\begin{array}{l}\text { Surveiller et maîtriser } \\
\text { les risques }\end{array}$ & $\begin{array}{l}\text { Le chef de projet doit procéder à la mise à jour des éléments de } \\
\text { l'actifet prendre en considération l'effet des demandes de } \\
\text { modification. }\end{array}$ \\
\hline
\end{tabular}

Tableau 5 Livrables PMBOK du Processus de Gestion des Risques

La figure suivante synthétise les principales techniques de chaque processus : 
*Audits des risques.

*Analyse de l'écart.

*Mesures de la performance.

*Analyse de la réserve.

*Réunions d'état du projet.

Stratégies pour les menaces.

* Stratégies pourles opportunités.

*Stratégies de réponse aux aléas.

*Jugement d'expert.

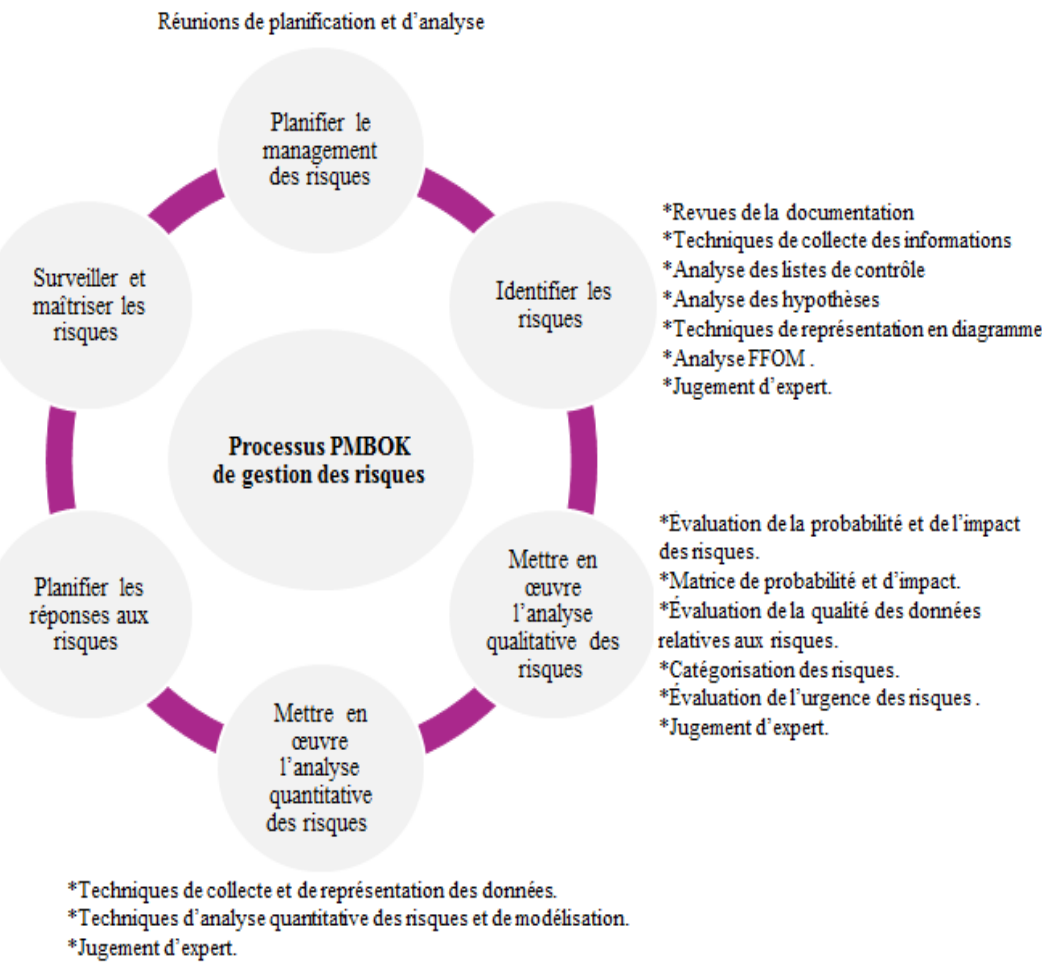

*Techniques d'analyse quantitative des risques et de modélisation.

*Jugement d'expert.

Figure 4 Principales Techniques PMBOK de Gestion des Risques

\section{Démarche de GP à mettre en place pour minimiser les risques d'échec :}

Cette démarche définit les principales actions qui doivent être entreprises lors de la conduite du projet, toutefois, elle ne garantit pas le succès global des projets, mais permet d'en sécuriser les exigences minimales de réussite.

Les étapes ou les actions loupées pourront être rattrapées et actées lors des ateliers de travail du lancement par les différents intervenants, l'expérience de ces derniers peut favoriser l'enrichissement de ladite démarche.Il est à préciser que cette démarche n'implique pas que les liens sont séquentiels, ils peuvent être lancés simultanément sous la responsabilité du directeur ou du sponsor du projet en fonction de la stratégie globale. 


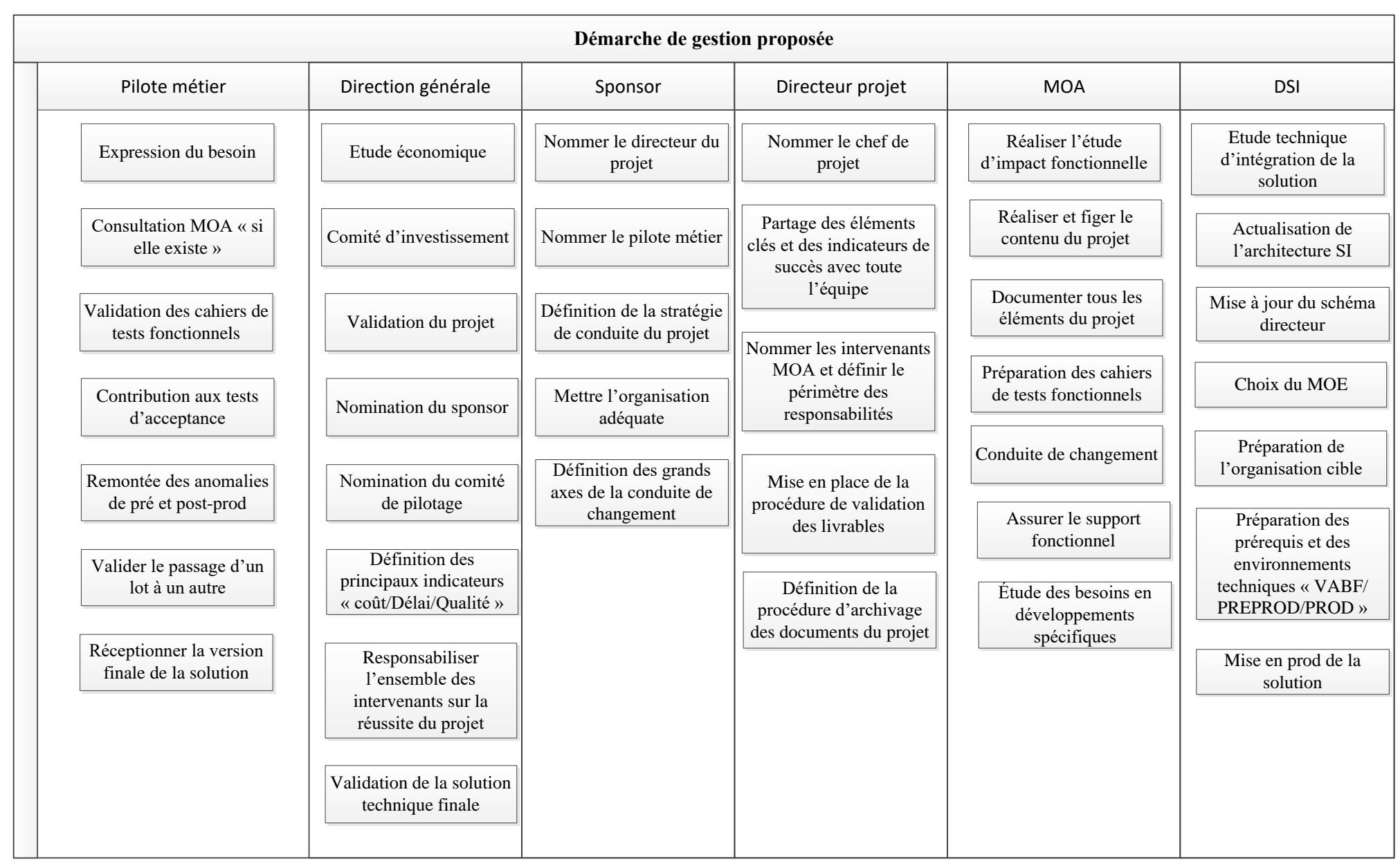

Figure 5 Démarche Proposée de Gestion des Projets ERP 


\subsection{Rôle des intervenants :}

\section{A. La direction générale :}

La DG doit définir une vision organisationnelle claire couvrant l'ensemble des volets du projet, ceci en se posant des questions relatives à la nécessité de recruter un sponsor, à l'organisation humaine à mettre en place ainsi qu'au recrutement des experts internationaux, elle doit ordonner la revue globale des règles fonctionnelles des processus ciblés par l'ERP.

Les exigences en matière d'expertise doivent être clairement définies, il s'agit de savoir s'il sera nécessaire de solliciter des chefs de projets jouissant d'une expérience assez significative et de compétences métiers actualisées ; ces compétences vont aussi déterminer la taille de l'équipe et impacter la nature et la quantité des interactions entre les différents intervenants.

Les principales actions sont retracées dans le logigramme suivant :

$$
\text { Rôle de la Direction Générale }
$$

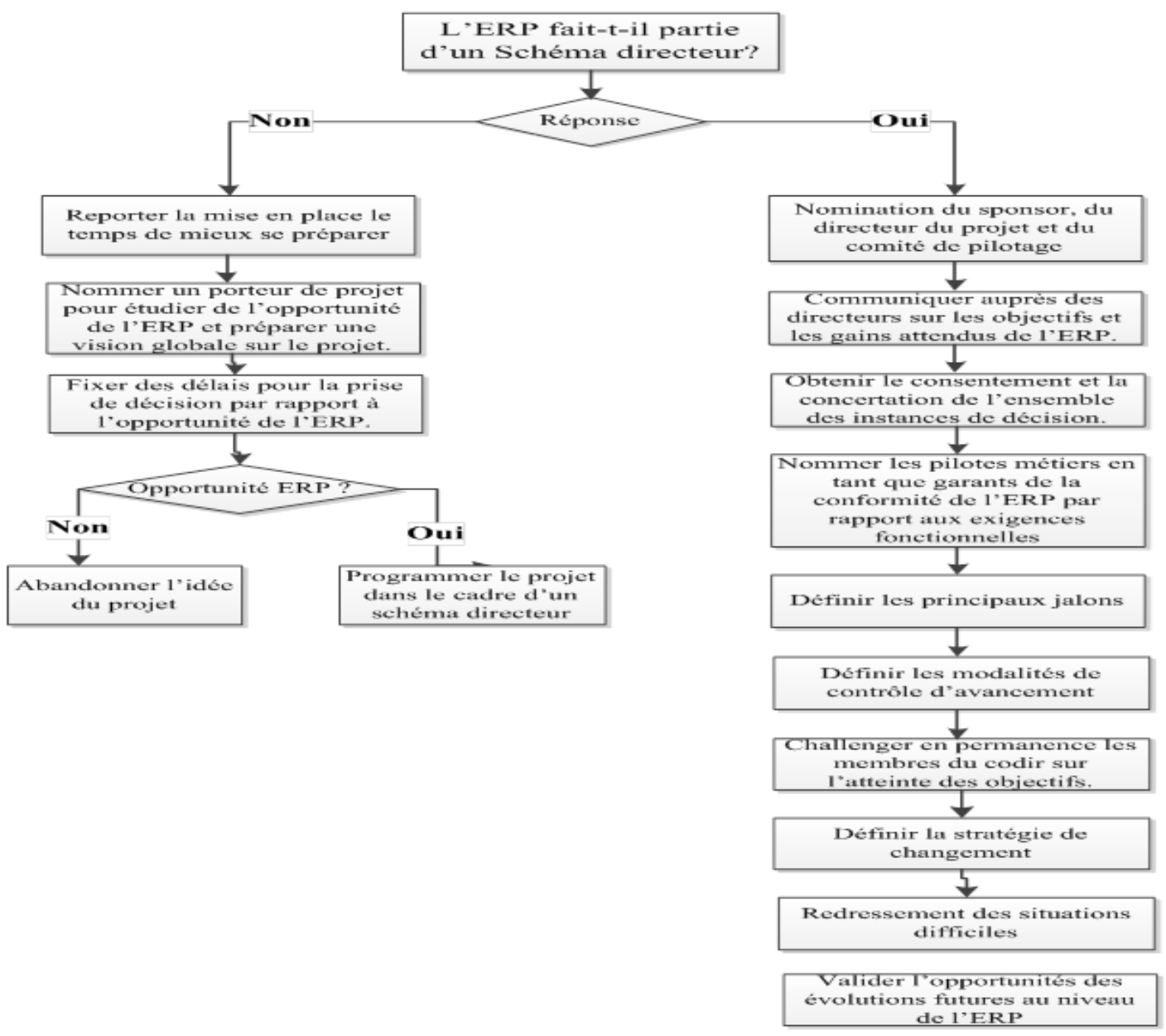

Figure 6 Rôle de la Direction Générale 


\section{B. Le Sponsor :}

Tout événement important ou projet d'ampleur nécessite la nomination d'un parrain dont le rôle est crucial pour la réussite du projet. Ce dernier, faisant partie du comité de pilotage, démontre l'intérêt de la Direction pour le projet et communique en interne sur l'objectif du projet ainsi que sur son avancement global, il procure ainsi une légitimité au projet.

De par sa crédibilité, son influence ainsi que sa position hiérarchique, il doit être vigilant en remontant tous les blocages critiques et assurer des arbitrages auprès de la direction générale quand ceci s'avère nécessaire. Il contribue à la conduite du changement sur tous les niveaux.

De par sa forte expérience, et son engagement envers l'organisation, le Sponsor exercera le rôle du patron. Il doit résoudre les sujets bloqués au niveau du chef de projet et assurer de même le support pour les situations conflictuelles.

Le sponsor de projet sera responsable des actions suivantes:

- Choix du directeur de projet.

- Définition des objectifs majeurs du projet.

- Evaluation de la performance du projet.

- Résolution des problèmes les plus bloquants.

- Valider les changements ayant le plus d'impact sur le contenu et sur les délais.

- Garantir le soutien et l'implication des différentes parties prenantes.

- Développer les bonnes pratiques de leadership au niveau de toute l'équipe.

- Garantir une communication fiable pour tout le projet.

- Approuver le passage d'un lot à un autre.

\section{La DSI :}

La DSI doit sécuriser la mise en place de la solution, elle agit sur plusieurs volets : 


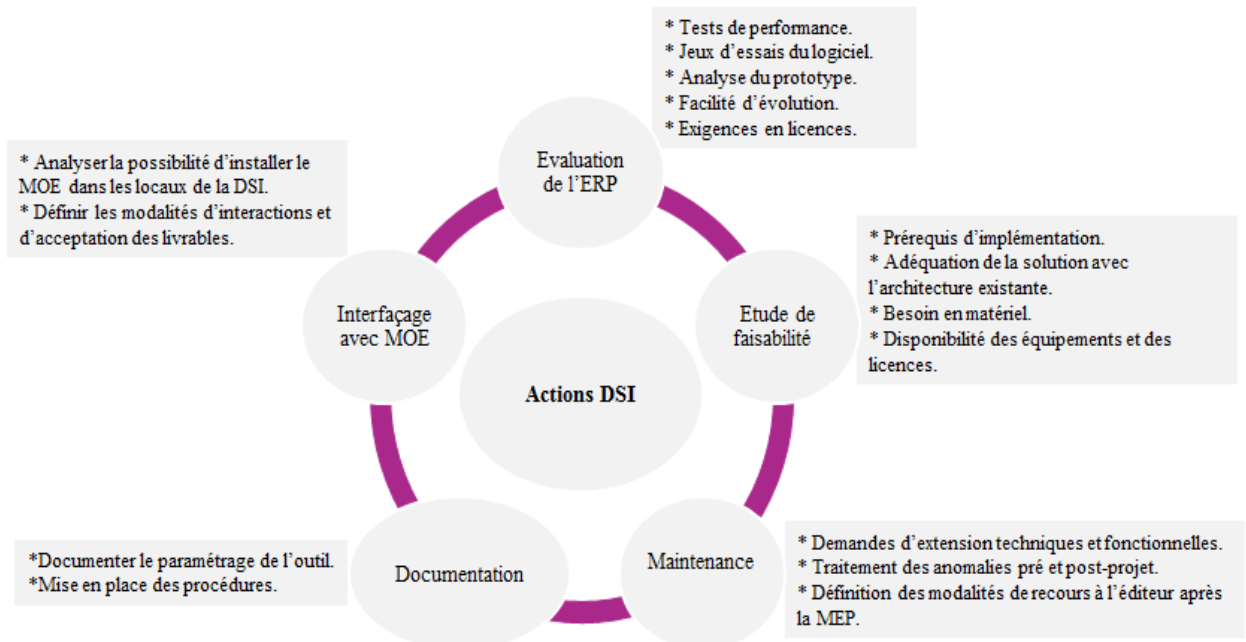

Figure 7 Responsabilités de la DSI

L'organisation de la DSI doit être discutée avec la MOA pour éclaircir les responsabilités, définir les mécanismes d'interactions et les modalités de remontée des alertes, elle est appelée à évoluer en fonction de la complexité du projet et des performances réalisées. De même, et à la différence du support fonctionnel MOA qui ne gère que les anomalies fonctionnelles post-prod, le support SI prend en charge les anomalies pré et post prod.

L'équipe exploitation doit assurer la mise en place de tous les prérequis techniques nécessaires pour une bonne exploitation du système, et en assurer les meilleures performances. Les actions d'amélioration, d'arrêt ou de MEP doit être faites en collaboration avec la MOA pour éviter tout impact sur les utilisateurs finaux.

L'organisation projet à mettre en place peut être comme suit :

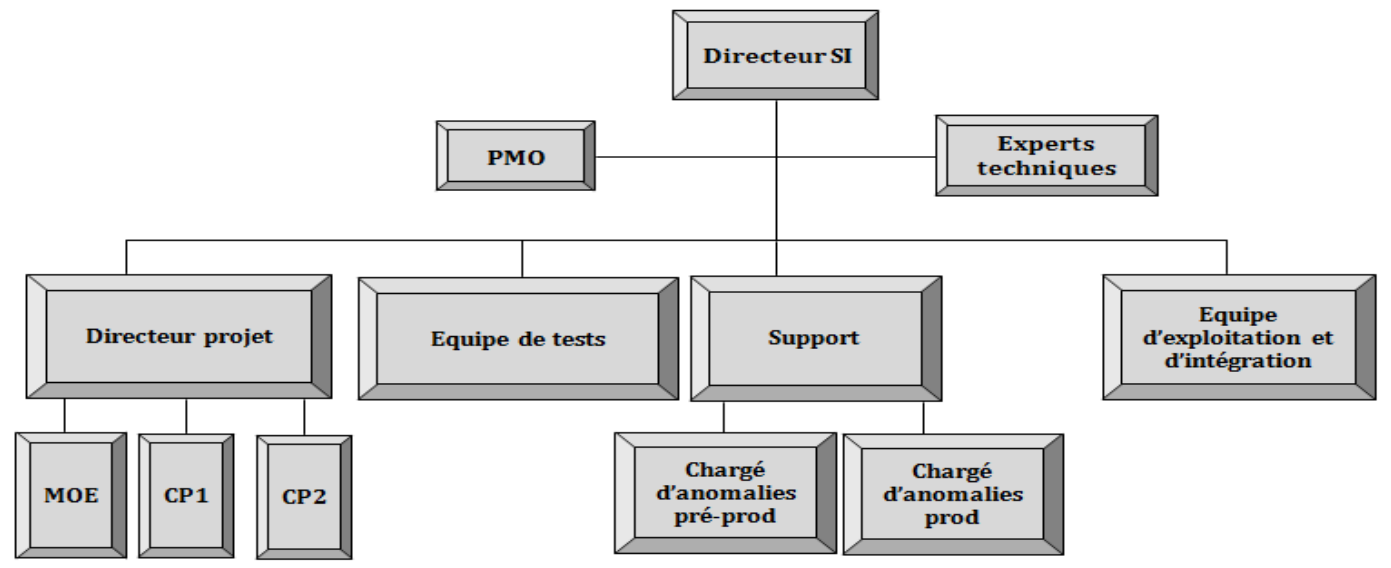

Figure 8 Organigramme de la DSI 


\section{Le comité de pilotage.}

Le soutien de la DG apparaît systématique pour garantir l'aboutissement du projet, le comité de pilotage doit ainsi définir une organisation adaptée au projet, il doit être composé de directeurs des différentes entités de l'entreprise, et ce, à l'instar de la direction financière, commerciale, achats, logistique et service clients.La participation de ces intervenants trouve sa justification dans l'utilité de l'ERP en question.

A travers des réunions de suivi hebdomadaires, bimensuelles ou mensuelles, le comité de pilotage analyse l'avancement du projet, prend les décisions adéquates à chaque situation et justifie tout éventuel dérapage à la DG.

Les actions et rôles du comité de pilotage s'articulent autour des volets suivants :

- Nomination du directeur de projet.

- Définition des modalités de suivi.

- Définition de la fréquence de suivi.

- Définition des principaux lots du projet.

- Nomination des membres du comité de crise.

- Sécuriser la disponibilité des prérequis du projet.

\section{E. Le directeur du projet}

Le directeur du projet, immédiatement après sa nomination, et avant le lancement du projet, doit entreprendre les dispositifs suivants:

- Constituer l'équipe du projet.

- Définition des parties prenantes.

- Délimiter le périmètre de responsabilité de l'équipe de projet.

- Partager les jalons du projet avec l'équipe.

- Organisation des workshops avec le métier.

- Préparer le cahier de charge.

- Etudier les offres techniques répondant au besoin exprimé.

- Soumission du CDC à la validation des entités impactées.

- Lancement de l'appel d'offre.

- Préparation de la grille d'évaluation fonctionnelle et technique de l'ERP.

Le cahier de charge ne doit être établi qu'après la tenue de workshop avec le métier pour exprimer le besoin fonctionnel, et non l'inverse, ceci pour éviter l'éventuelle insatisfaction de l'utilisateur final. De même, le directeur doit analyser l'opportunité de nomination d'un PMO pour garantir un meilleur suivi de projet et déclencher les plans d'actions adéquats aux différentes situations.L'évaluation doit prendre les critères suivants en considération : 
Evaluation technique: Elle est sous la responsabilité du directeur du projet et du directeur des systèmes d'informations qui doivent définir et étudier leur stratégie, ainsi que leurs tactiques organisationnelles relatives à la nature de la solution qui sera implémentée, et aussi par rapport à l'état des lieux des systèmes existants et de l'architecture technique mise en place et des exigences en matière de licence.

Evaluation fonctionnelle: A travers l'ergonomie du système et la simplicité de son utilisation, la nature des processus et les transactions supportées par la solution, la fiabilité et la sécurité des accès, la fiabilité des rapports ainsi que l'interfaçage avec les autres systèmes existants.

Délais de livraison: Les délais de livraison sont très importants dans les cas où l'implémentation de l'ERP conditionne la stratégie commerciale de certaines entreprises de services; ceci trouve sa justification dans l'acharnement de la concurrence.

Evaluation financière: Qui a pour objectif de déterminer le coût des différentes solutions proposées par les soumissionnaires, d'analyser la conformité par rapport au budget initial alloué, de négocier les conditions de paiement ainsi que les différentes modalités contractuelles relatives aux pénalités....

Evaluation support: Relative aux coûts de maintenance, ainsi que la nature des prestations de support. A cet effet, deux scénarios existent : Le premier consiste en la formation de collaborateurs internes ainsi que leur accompagnement pour développer les compétences relatives à la gestion des anomalies du système. Le deuxième consiste à faire un contrat de maintenance avec l'intégrateur de la solution.

Références de l'éditeur: la renommée de l'éditeur, son image de marque, sa capacité de financement et les projets réalisés sont un facteur déterminant de sélection du fournisseur.

\section{F. Rôle du pilote métier.}

Le pilote métier s'assure de la cohérence des livrables par rapport aux spécifications et aux exigences initiales, Il se porte garant des objectifs opérationnels de son activité et de l'efficience des processus. Sa contribution doit se dérouler tout au long des étapes de réalisation du projet, il ne doit nullement assumer les conséquences de la mise en production d'un outil qui ne répondra pas aux exigences fonctionnelles des processus de son activité. Dans cette démarche proposée, le rôle du pilote métier est l'un des plus impactant pour la réussite du projet. Son intervention ne doit pas se limiter à l'utilisation du système après sa mise en production, mais le dépasser à un rôle de consulting, d'assistance du chef de projet, ainsi que la validation et la réception finale de l'outil. Le pilote métier doit agir dans l'ensemble des étapes de préparation, de conduite et d'exploitation de l'outil comme suit : 


\begin{tabular}{|l|l|}
\hline Etape & Actions \\
\hline \multirow{2}{*}{ Pré-projet } & $\begin{array}{l}\text { Validation du périmètre du projet et du contenu global de l'outil. } \\
\text { Validation des délais de mise en production pour adaptation au changement. }\end{array}$ \\
\hline \multirow{3}{*}{ Conduite } & $\begin{array}{l}\text { Validation du passage d'un lot à un autre. } \\
\text { Validation des livrables. } \\
\text { Validation de la qualité des livrables. } \\
\text { Analyse des performances de l'outil sur l'environnement préprod. }\end{array}$ \\
\hline \multirow{3}{*}{ Exploitation } & $\begin{array}{l}\text { Utilisation de l'outil. } \\
\text { Remontée des anomalies. } \\
\text { Elaboration des demandes d'évolution supplémentaires. }\end{array}$ \\
\hline
\end{tabular}

Tableau 6 Responsabilités du Pilote Métier

Les actions du pilote métier lors de l'étape «pré-projet» vont permettre de figer le contenu, et éviteront ainsi leschangements ou les besoins mal exprimés. De même, ces actions lors des autres étapes contribueront aux performances globales du projet.

\section{G. Rôle de la MOA.}

La MOA assure l'organisation de la conduite du projet, en assure l'efficacité et optimise les, l'organisation à mettre en place peut être comme suit :

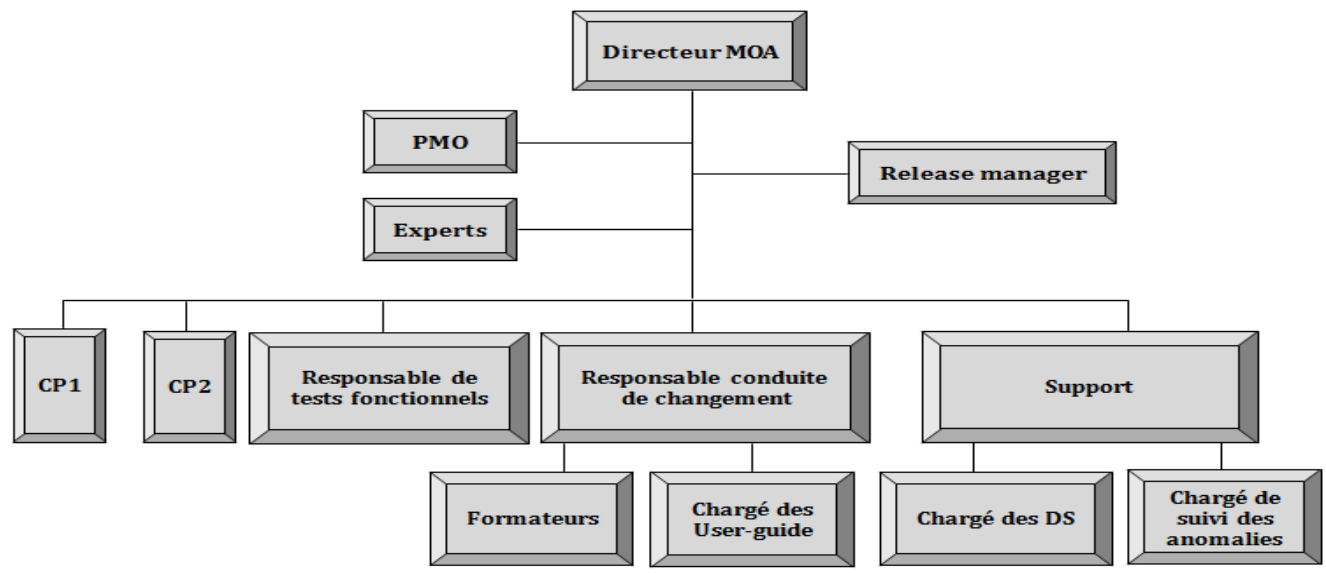

Figure 9 Organigramme de la MOA

Les périmètres de responsabilité de chaque intervenant doivent être clairement définis pour éviter l'apparition des conflits ; ils s'articulent autour des points suivants :

- L'équipe de tests déroule les tests d'acceptance des nouveaux livrables, ainsi que les tests de non régression des anciennes fonctionnalités.

- La conduite de changement assure les formations et la rédaction des user-guide. 
- Les chefs de projets sont rattachés hiérarchiquement au directeur de la MOA, et fonctionnellement au PMO.

- Le release manager assure l'interfaçage avec la DSI pour organiser les livrables.

- Les experts assisteront le directeur sur certains volets.

- Le PMO assurera le suivi d'avancement, pour consolider les données, pour dresser des plans d'actions et en assurer le suivi, et remonter les alertes.

- Le directeur déclinera les objectifs stratégiques en objectifs opérationnels.

Le support fonctionnel doit disposer d'une maitrise $\mathrm{du}$ fonctionnement du système informatique, le périmètre de ses responsabilités s'articule autour des points suivants :

- Assister les entités métier pour l'utilisation de l'outil en cas de difficultés.

- Piloter la procédure de gestion des accès à l'outil.

- Valider les user-guide de l'outil.

- Analyser les anomalies remontées par les utilisateurs.

- Coordonner la résolution des anomalies entre la DSI et le métier.

- Mise en place des solutions de contournement des anomalies.

- Mise en place d'une base de connaissances d'incidents et d'anomalies.

- Proposer des plans d'amélioration continue.

Le support fonctionnel doit formaliser la procédure de traitement des anomalies fonctionnelles, il doit proposer les actions de contournement pour enréduire l'impact. De même, il prend en charge la procédure de gestion des droits d'accès au système.

\section{H. Le chef du projet :}

Les actions à entreprendre par le chef de projet s'articulent autour des points suivants :

- Rédaction et validation de l'expression du besoin.

- Organiser des ateliers avec le métier et la MOE.

- Remontée des alertes et mise en place de plans d'actions.

- Suivi des jalons et validation des livrables.

- Analyse de l'impact des modifications.

- Elaborer le reporting global.

- Communiquer sur l'avancement du projet.

- Gérer les risques.

- Challenger la MOE sur l'atteinte des objectifs. 


\subsection{Démarches de contrôle et suivi des performances :}

\section{A. La stratégie du métier et impact organisationnel de l'ERP.}

Lors de la mise en place de l'ERP, l'entreprise vit un véritable changement technico-organisationnel, le métier doit adopter des dispositifs organisationnels pour assurer une utilisation efficace de l'outil. Les éléments suivants doivent être pris en considération :

- Périmètre de responsabilité de chaque intervenant.

- Les changements d'attributions et de rôles ?

- Comment éviter la dépendance vis-à-vis des collaborateurs ?

- Doit-il y avoir une spécialisation ou polyvalence des collaborateurs par domaine?

- Maitrise du système par les collaborateurs?

- Comment évaluer le degré de satisfaction des utilisateurs par rapport à l'ERP.

Il est conseillé de créer une cellule pour assister les utilisateurs et apporter des solutions par rapport aux problèmes rencontrés au quotidien ; il s'agit d'entité portant la dénomination de «Helpdesk» ou "centre de compétences » composé au minimum de deux collaborateurs, ces derniers interviennent en tant que SPOC vis-à-vis des utilisateurs et de la DSI.

\section{B. La conduite de changement.}

Après la finalisation des travaux de développement, l'implémentation de l'ERP ne sera efficace que s'il y'a adhésion de tous les utilisateurs finaux, même si cela implique le changement des méthodes de travail.Au-delà du choix de la solution, la direction générale doit aussi intervenir lors de la mise en production; une équipe spécialisée en conduite de changement et en support doit être mise en place.

L'un des points importants auxquels il faudra prêter une attention particulière est la gestion de la complexité, en effet ; Plus l'ERP sera utilisé au niveau national, plus son déploiement sera plus complexe.A cet effet, la MOA doit sensibiliser les futurs utilisateurs sur les bénéfices attendus de la mise en place de l'ERP par rapport au déroulement des opérations quotidiennes, qu'ils soient relatifs au gain de temps, à la simplification des tâches ainsi qu'à l'optimisation des processus, elle doit assurer une formation pédagogique basée sur l'utilisation des différents modules de l'outil, et fournir tous les supports nécessaires à l'accompagnement.

Les problèmes qui risquent d'être rencontrés et qui doivent être surmontés par le responsable de conduite de changement sont les suivants :

1- Manque d'intérêt des utilisateurs.

2- Démotivation des différents acteurs.

3- Présence de sentiment d'insécurité et d'inconfort. 
4- Forte réticence au changement avec risque de rejet total du système.

5- Perte de repères de la part des utilisateurs.

6- Insuffisance des compétences nécessaires à l'utilisation de l'ERP.

\section{Suivi d'avancement et des performances.}

$\mathrm{Vu}$ l'aspect dynamique du contexte dans lequel se réalisent les projets ERP, l'évolution doit être mesurée en permanence, ceci afin de prendre les décisions les plus appropriées. L'avancement du projet doit être fait à l'aide d'indicateurs qui peuvent être classifiés en :

- Indicateurs de processus.

- Indicateurs de résultats.

- Indicateurs d'effets collatéraux ${ }^{\mathbf{4 0}}$.

Le nombre d'indicateurs de suivi du projet doit être restreint au minimum pour en faciliter le contrôle; on pourrait retenir d'après les experts, à titre indicatif, entre 5 et 10 indicateurs.

L'avancement global de chaque lot du projet doit se faire sur la base du diagramme de GANTT, celui-ci facilite le suivi du projet et permet de constater rapidement les ajustements à apporter et de vérifier si l'utilisation des ressources concorde avec ce qui avait été planifié, le chef de projet pourra présenter le modèle ci-dessous pour chaque lot :

\begin{tabular}{|c|c|}
\hline Réalisation week précédent & Prochaine étape \\
\hline & \\
\hline Risques et anomalies bloquantes & Actions de réduction des risques \\
\hline & \\
\hline
\end{tabular}

Tableau 7 Modèle de Suivi Du Projet

Il est à préciser que le choix du modèle de suivi dépend de plusieurs facteurs à l'instar de la taille du projet, de l'expérience des intervenants, ainsi que des outils de reporting disponibles.

\subsection{Démarche de gestion des risques :}

Les intervenants au niveau de ce volet sont :

40Requiert une analyse prospective des impacts indésirables associés à l'implantation du projet. 


\begin{tabular}{|l|l|}
\hline Intervenant & Nature de l'apport \\
\hline Chef de projet & Pilote la gestion des risques. \\
\hline Directeur du projet & Valide le plan et les stratégies de gestion des risques. \\
\hline Pilote métier & $\begin{array}{l}\text { Aide à l'identification des risques, et prépare son entité aux impacts des } \\
\text { risques éventuels. }\end{array}$ \\
\hline Parties prenantes & Recensement des risques. \\
\hline Experts & Apport d'expertise sur les volets identification et traitement des risques. \\
\hline Direction générale & Validation de la stratégie de gestion et allocation des moyens. \\
\hline
\end{tabular}

Tableau 8 Apport des Intervenants en Gestion des Risques

L'enchainement des étapes pour une gestion efficace des risques est comme suit :

a. Préparation de la $\mathrm{SDP}^{41}$ détaillée.

b. Tenue de réunions de brainstorming avec les différentes parties prenantes pour identifier et préparer les actions de traitement des risques.

c. Recensement des risques identifiés.

d. Préparation du canevas et de l'outil de suivi comme suit :

\begin{tabular}{|l|l|l|l|l|l|l|}
\hline $\mathrm{N}^{\circ}$ & Lot impacté & Nature risque & Descriptif & Probabilité & Gravité & Descriptif \\
\hline $\mathrm{R} 1$ & & & & & & \\
\hline $\mathrm{R} 2$ & & & & & & \\
\hline
\end{tabular}

e. Partage et validation de la liste avec l'ensemble des intervenants.

f.Tenue de réunion avec les experts pour mise en place du plan d'action adéquat.Le tableau suivant doit être mis en place :

\begin{tabular}{|l|l|l|l|l|l|}
\hline $\mathrm{N}^{\circ}$ & $\begin{array}{l}\text { Action de } \\
\text { réduction }\end{array}$ & $\begin{array}{l}\text { Action } \\
\text { d'élimination }\end{array}$ & $\begin{array}{l}\text { Action de } \\
\text { contournement }\end{array}$ & $\begin{array}{l}\text { Responsable } \\
\text { contrôle }\end{array}$ & $\begin{array}{l}\text { Modalités } \\
\text { de contrôle }\end{array}$ \\
\hline $\mathrm{R} 1$ & & & & & \\
\hline $\mathrm{R} 2$ & & & & & \\
\hline
\end{tabular}

g. Mise en place d'une procédure de crise pour décrire la nature des actions à entreprendre en cas de concrétisation des risques identifiés, et définir de même les modalités de remontée et d'escalade des alertes.

h. Définir les modalités de suivi et de contrôle des risques.

i. Validation du plan d'actions par le directeur.

j.Soumission de la stratégie de gestion des risques à la validation de la direction générale.

41Structure de découpage du projet. 


\subsection{Organisation des interactions entre intervenants.}

La réussite se joue au niveau de l'efficacité des interactions entre intervenants, les intérêts globaux doivent dominer sur les intérêts personnels. En tant que bonne pratique, la mise en production doit se dérouler en présence de la recette MOA, du support fonctionnel et des équipes DSI ; les tests de non régression et de conformité doivent être déroulés. En cas de survenue d'anomalies, la situation doit être analysée par les décideurs pour prise de décisions par rapport à un éventuel roll-back.

\section{Conclusion générale}

Quelle que soit la nature du projet et l'environnement dans lequel il est réalisé, le chef de projet doit identifier, de manière la plus exhaustive possible, l'ensemble des événements susceptibles de générer des risques fortement impactant pour les objectifs du projet et les classer par nature, qu'ils soient d'ordre organisationnel, politique, commercial ou technique. Cette identification doit être suivie d'une analyse minutieuse de leurs sources ainsi que de leurs éventuelles incidences.

La gestion de ces risques consiste à mettre en œuvre les actions adéquates pour les contrôler et en assainir l'impact, soit en supprimant les causes, soit en le transférant à une autre partie-prenante. Une autre stratégie consiste à accepter le risque tout en le contrôlant minutieusement. Il convient ensuite au chef de projet de mettre à jour le portefeuille des risques en fonction des évènements surgis et d'en revoir la criticité et les composantes au fur et à mesure de l'avancement du projet, Il est judicieux de procéder régulièrement au suivi et au contrôle desdits risques.

\section{References:}

\section{Ouvrages:}

BillBelt. (2008), Les basiques de la gestion industrielle et logistique. Eyrolles. Editions d'organisation.

Christian Dumont (2007): ITIL pour un service informatique optimal 2ème édition.Eyrolles.

Daniel Tixier, Hervé Mathe et Jacques Colin. (1996), La logistique d'entreprise Vers un management plus compétitif. DUNOD.

Dominique Moisand. (2009):COBIT Pour une meilleure gouvernance des systèmes d'information.Eyrolles.

Michel Winter. (2013), Gestion de projet en SSII.Ellipses.

Project Management Institute. (2008), Guide du Corpus des connaissances en management de projet, Quatrième édition. PMI.

Rémy LeMoigne. (2013), Supplychain management: Achat, production, logistique, transport, vente. DUNOD. 
The Stationery Office. (2009), Directing Successful Projects with PRINCE2 Edition 1. Office Of Government Commerce.

Yves Pimor, Michel Fender. (2008), Logistique : production, distribution, soutien, 5ème édition. DUNOD.

\section{Articles:}

Amel CHAABOUNI. (2006), IMPLANTATION D'UN ERP: ANTECEDENTS ET CONSEQUENCES. XVème Conférence Internationale de Management Stratégique, Annecy / Genève 13-16 Juin 2006.

Aurélien ROUQUET, Pascal LIEVRE. (2008), Management logistique et outils de gestion : un dialogue nécessaire.

Werner DELFMANN, Martin GEHRING. (2003), Le rôle des technologies de l'information dans la performance logistique.revue Logistique \& Management

Marie-France HALLÉ, Jacques RENAUD, Angel RUIZ. (2005), Progiciels de gestion intégrée : Expériences d'implantation dans cinq entreprises Québécoises.

Thi Le Hoa VO, Laurent BIRONNEAU. (2011),Systèmes d'information et gestion globale de la chaine logistique : un état de l'art.

Laurent LIVOLSI, Nathalie FABBE-COSTES. (2003), La centralité des systèmes d'information (S.I.) dans la fonction logistique: validation empirique et interrogations sur l'impact d'Internet.

Laurent ARCIS. (1999), L'impact des ERP sur la chaîne logistique.

François Meyssonnier, Frédéric Pourtier. (2004), ERP, changement organisationnel et contrôle de gestion.

IyadZoukar, Camille Salinesi, Colette Rolland. (2012), Evolution du système d'information par l'implantation d'un Progiciel de Gestion Intégrée: Systématiser la mise en correspondance entre le système et l'organisation

\section{Rapports :}

Pôle interministériel de prospective et d'anticipation des mutations économiques en France. (2009), L'impact des technologies de l'information sur la logistique

Jean-Grégoire Bernard, Suzanne Rivard, Benoît Aubert. (2002), Evaluation du risque d'implantation de progiciel. 